\title{
LXIII.
}

Aus der II. med. Klinik zu Berlin.

\section{Ueber protoplasmatische Körperchen in den Lymphdrüsen Syphilitischer ${ }^{1}$ ).}

Von

\author{
Dr. Paul Reckzeh,
}

klin. Assistenten.

(Hierzu Tafel Xx nucl 1 Fig. im Text.)

Die in neuester Zeit erfolgten Veröffentlichungen über Syphiliserreger haben das Interesse der Untersucher auf die Mikroskopie syphilitischer Gewebe gelenkt. Der Bericht von Schaudinn und Hoffmann²) über das Vorkommen von Spirochaeten in syphilitischen Krankheitsproducten hat die hier mitzutheilenden Untersuchungen, welche zunächst eine Nachuntersuchung der Schaudinn-Hoffmann'schen Befunde bezweckten, veranlasst.

Die von den genannten Autoren im syphilitischen Gewebe gefundene Spirochaete (Spirochaete pallida) unterscheidet sich von den zahlreichen, im Mund und an den Genitalien oberflächlich vorkommenden Spirochaeten (Spirochaete refringens u. A.) dureh die Kleinheit und Zartheit, ihr geringes Lichtbrechungsvermögen, die (korkzieherartige) Art ihrer spiraligen Aufwindung und die sehr geringe Färbbarkeit.

Jch habe bei meinen Nachuntersuchungen die Spirochaete pallida in ausserordentlich zahlreichen Fällen von Lues ${ }^{3}$ ), dagegen niemals bei anderen Erkrankungen finden können. Ueber einschlägige Blutuntersuchungen habe ich an anderer Stelle berichtet.

1) Nach einem Vortrage in der Gesellschaft der Charité-Aerzte vom 2. November 1905 .

2) „Vorläufiger Bericht über das Vorkommen von Spirochaeten in sypbilitischen Krankheitsproducten und bei Papillomen". Arbeiten aus dem Kaiserl. Gesundheitsamte. Bd. XXII. H. 2. 1905.

3) Das Material wurde mir mit gütiger Eriaubniss des Herrn Prof. Dr. Lesser von Herrn Privatdocent Dr. Hoffmann und den Herren Stabsärzten Dr. Roscher und Dr. Grumme freundlichst zur Verfügung gestellt, wofür ich an dieser Stelle verbindlichst danke. 
Inzwischen haben Schaudinn und Hoffmann ${ }^{1,2, ?, 4}$ ) selbst ibre Untersuchungen auf ein sehr umfangreiches Material, auch bei hereditärer Lues, ausgedehnt, und es sind über 100 Mittheilungen über Nachuntersuchungen erschienen, welche in der überwiegenden Mehrzahl ihre Befunde bestätigen. Besonders umfangreiche Controluntersuchungen wurden von Roscher angestellt.

Ich habe nun zunächst den durch Punction mittels einer starken Spritze gewonnenen Leistendrïsensaft von 25 Syphilitischen untersucht und zwar in frischen Präparaten und nach Färbung mit Borax-Methylenblau, Triacid, Romanow ski'scher Eosin-Methylenblaulösung und Giems ascher Mischung; von letzterer giebt man am besten 15 Tropfen auf $10 \mathrm{ccm}$ Aqua destill. und färbt in Blockschälchen nach halbstündiger Alkohol-Aetherfixation ctwa eine Stunde. Ioh habe bei sämmtlichen Fällen von Iules neben den normalen Drüsenelementen eigenthümliche, protoplasmatische Körperchen gefunden. Dieselben waren auch Hoffmann in seinen Präparaten aufgefallen.

Normaler Weise findet man im Drüsensaft zahlreiche, kleine und grosse Lymphocyten, polynukleäre, neutrophile Leukocyten, rothe Blutkörperchen und die übrigen Elemente des Blutes, Lymphdrüsenparenchymzellen und freie Zellkerne. Bei den Fällen von Lues sieht man ausserdem neben zahlreichen Exemplaren der Spirochaete pallida kleine, meist. kreisrunde Körperchen, von denen viele wie Zellen aussehen. Ihre Grösse schwankt zwischen der eines Blutplättchens und eines Lymphocyten, ihre Anzahl ist oft bedeutend grösser als die der normalen Drüsenelemente. Oft ist die Randzone ein wenig stärker gefärbt als die Innenzone. Eigene Beweglichkeit fehlt den Körperchen, wie die Betrachtung frisch entnommenen Materiales beweist. Färberisch verhalten sic sich genau wie das Protoplasma der Zellen (siehe Abbildung 1).

Diese Körperchen sah ich in geringerer Anzahl auch im lymphadenoiden Gewebe anderer Organe und zwar anch bei einem Falle von hereditärer Lues, dessen Präparate mir Herr College Hoffmann freundlichst zur Verfügung stellte; ich vermisste sie oder fand sie höchstens vereinzelt bei Drüsenschwellungen aus anderen Ursachen. Untersucht wurden daraufhin Tuberculose, lymphatische und gemischtzellige Leukämie, normale Drüsen, Ulcus molle, Gonorrhoe, Balanitis. Wenn es sich also auch nicht um einen für Lues specifischen Befund handelt, so ist vielleicht ihr gehäuftes Vorkommen bei Lues doch von einigem differentialdiagnostischen Werth.

1) „Ueber Spirochaete pallida bei Syphilis und die Unterschiede dieser Form gegenüber anderen Arten dieser Gattung." Berliner klinische Wochenschrift. 1905. No. 22, 23.

2) „Ueber Spirochaetenbefunde im Lymphdrüsensaft Syphilitischer". Deutsche med. Wochenschr. 1904. No. 18.

3) Hoffmann, "Weitere Mittheilungen über das Vorkommen der Spirochaete pallida bei Syphilis". Berliner klin. Wochenschr. 1905. No. 32.

4) Hoffmann, „Ueber die Spirochaete pallida". Deutsche med. Wochenschr. 1905. No. 43. 
Es fragt sich nur, woher die geschilderten Körperchen stammen. Nach ihrem färberischen Terhalten sind sie protoplasmatischer Herkunft. Dass die Lymphocyten, an denen schon Ehrlich eine Auffaserung des Protoplasmasaumes beschrieben hat, nicht die Veranlassung ihrer Bildung sind, geht daraus hervor, dass man fast immer ausserordentlich zahlreiche Körperchen neben meist wohlerhaltenen Lymphocyten findet. Es ist also wahrscheinlich, dass die Körperchen aus den viol fragileren Drüsenparenchymzellen bei deren Zerfall entstehen. Dass viele solche Zellen zerfallen, geht aus der grossen Anzahl ibrer Kerntrümmer hervor. Zuweilen sieht man noch ziemlich erhaltene solche Zellen, an denen sich Theile des Protoplasmas abschnüren, die dann durch mechanische Verhältnisse eine runde Gestalt bekommen können. Noch mehr spricht für die Annahme dieser Entstehungsart der Befund von kleinen Schollen auf den Körperchen, welche genau die Färbung der Kerne der Drüsenparenchymzellen aufweisen, also wolll dem Protoplasma anhaftende Kerntrümmer sind. Bei der Giemsa-Färbung sieht man die Körperchen hellblau, z. T. mit röthlichen Punkten besetzt. Dass gerade bei der Lues sich so auffallend viel Zerfallsproducte der Zellen finden, ist merkwürdig. Vielleicht spielt da eine specifische Giftwirkung eine Rolle.

Ueber diese Lymphdrüsenparenchymzellen bezw. das Lymphdrüsen gewebe hat nicht immer Uebereinstimmung geherrscht. Während man früher (Bizzozero) annahm, dass die Drüsen aus einem reinen Fasernetz beständen, welchem fixe Zellen, die "Reticulum-Endothelien", nur angelagert seien, beschrieben spätere Untersucher ausserdem noch Zellen, welche mit den Fasern in directer Verbindung stehen. Nach $0 \operatorname{rth}^{1}$ ) zeigt das Reticulum der Drüsen eine zellige Struetur; allmälich schwinden dann die Zellen und ihre Ausläufer bleiben als zarte, nicht mit Bindegewebe identische Balken übrig. Nach Untersuchungen von R. Thomé2) besteht das Reticulum der Lymphknoten (Sinus nnd Parenchym) aus anastomosirenden Zellen, von denen ein Theil zu bindegewebeähnlichen Fasern differenzirt ist, die innerhalb des Protoplasmas liegen und wie dieses Netze bilden. Bei alten Leuten findet man in den Zellausläufern ausser diesen Reticulumfasern auch elastische Fasern. In fast allen Schnitten jedoch, besonders in den Drïsen von Kindern, finden sich Reticulumbälkchen, welche keine Fasern enthalten. Die sogenannten Endothelzellen sind nur plattgedrückte Reticulumzellen. Einen gleichen Bau zeigen Kapsel und Trabekel der Drüsen.

Eine derartige Reticulumzelle mit eingelagerten Fasern, von einer jungen Katze stammend, möge die, umstehende Figur $^{3}$ ) veranschaulichen.

Mit den übrigen, im Blut und Gewebssaft Syphilitischer beschriebenen Gebilden sind die hier charakterisirten Körperehen aus morphologischen und färberischen Gründen nicht identischl.

1) "Cursus der normalen Histologie". 3. Aufl. Berlin 1884.

2) Beiträge zur mikroskopischen Anatomie der Lymphknoten. Jenaische Zeitsehrift f. Naturwissenseh. Bd. 37. 1902, S. $134 \mathrm{ff}$.

3) Nach T'homé l. c. 


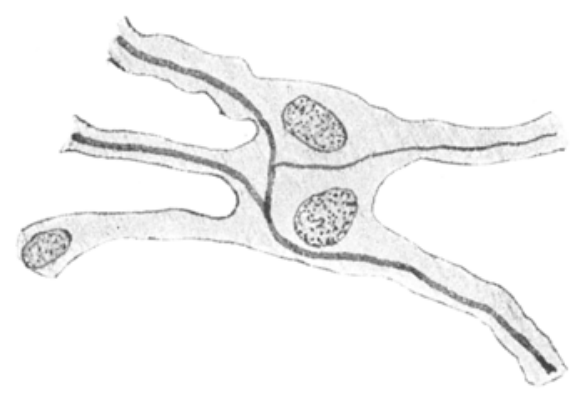

Im Jahre 1872 beschrieb Lostorfer ${ }^{1}$ ) in Wien im Blute 'Syphilitischer gewisse Körperchen, welche cr Sypliliskörper nannte. Er brachte einen Tropfen Blut in die feuchte Kammer und sah vom 3. bis 5. Tage an kleine, glänzende Körperchen, die zum Theil schwingende Bewegungen ausführten und von denen einige einen kleinen Fortsatz zeigten. An den folgenden Tagen nahm ihre Grösse, Zahl und die „Sprossenbildung“" zu. Viele der Körperchen wurden grösser als ein rothes Blutkörperchen und zeigten eine unregelmässige Gestalt. Nach dem 8. bis 10. Tage bildete sich in den Körperchen cine Vacuole, womit die Entwickelung der Gebildo ihr Lnde erreichte. Zuweilen entwickelten sich die Körperehen, von denen Lostorfer einmal 50 in einem Gesichtsfelde sah, rascher oder anch langsamer. In dem gleichfalls untersuchten Blut von Diphtherischen, Gonorrhoischen und Ekzematösen wurden die Körperchen vermisst? Wedl ${ }^{2}$ ) erklärte die von Lostorfer beschriebenen Körperchen für Fett, und zwar Fett, welches als zufällige Verunreinigung von der Haut in das Blut kam. In der an diese Mittheilungen sich anschliessenden Discussion beschrieben dann andere Untersucher ähnliche Körperchen auch für andere Krankheiten. Ich habe ähnliche Körperchen wie die Lostorfer'schen weder im Blut noch im Gewebssafte je finden können.

Im Jahre 1898 beschrieb dann F. Winkler in Wien Körperehen in syphilitischen Producten, welche er als "tingible Kugeln" bezeichnete. Er fand in syphilitischen Gewebsschnitten bei der Färbung mit wässerigalkoholischen Lösungen von Thionin oder Toluidinblan und Entfärbung mit Formalin theils frei liegende, theils in Zellen eingeschlossene Körperchen, welche meist kleiner als die Zellkerne waren, einen hellen Innenfleck zeigten und durch einen helleren Hof von den übrigen Gewebszellen getrennt waren. Aehnlichen Gebilden begegnete er allerdings auch in Schnitten von Lupus vulgaris, welche er in gleicher Weise behandelte. Winkler ${ }^{3}$ ) hält diese Körperchen für nicht identisch mit den von

1) Ueber die Möglichkeit der Diagnose derSyphilis mittelst der mikroskopischen Blutuntersuchung. Arch. f. Dermat. u. Syph. 1872. S. $115 \mathrm{ff}$.

2) Arch. f. Dermat. u. Syph. 1872. S. $124 \mathrm{ff}$.

3) Ueber tingible Kugeln in syphilitischen Producten. Arch. f. Dermat. u. Syph. 1898. S. $3 \mathrm{ff}$. 
Ueber protoplasmatische Körperchen in den Lymphdrüsen Syphilitischer. 653

Flemming ${ }^{1}$ ) in den Keimcentren der Lymphdrüsen beschriebenen tingiblen Körpern, welche ebenfalls keine Structur erkennen lassen, oder mit den ähnlichen Gebilden, welche Hindenburg ${ }^{2}$ ) in leukämischen Organen beschrieb. Mit den Russell'schen Körperchen sind die Gebilde ebenfalls nicht zu verwechseln, da die ersteren oft in Haufen zusammenliegen und niemals einen "Innenfleck" aufweisen. Winkler hält es für möglich, dass die von ihm beschriebenen Körperchen aus zusammengeflossener Chromatinsubstanz gebildet werden, welche aus den Zellen auf dem Wege der Lymphbahn in die Gewebe transportirt wird. Er neigt der Meinung zu, dass ,es sich um eine Kernerkrankung handle, welche unter dem Einflusse des syphilitischen Virus zu Stande kommt, die aber, wenn auch in geringerem Maasse, auch durch andere Virusarten (Lupus) bewirkt werden kann." (Siehe Abbildung II).

Von diesen "tingiblen Kugeln" unterscheiden sich die Körperchen durch ihr färberisches Verhalten und dadurch, dass sie eine deutliche Structur aufweisen.

Ferner hat Döhle ${ }^{3}$ ) 1892 im Schankersecret und im Gewebssaft von congenital Syphilitischen, dann auch im Blute $\left.{ }^{4}\right)$ Protoplasmagebilde gefunden, die er als parasitäre Protozoen ansprach. Aehnliche Formen (siehe Abbildung II) habe ich niemals gefunden.

Der Cytorrhyctes luis Siege ${ }^{5}$ ) endlich, von welchem verschiedene Formen (Birnen-, Walzen-, Hantelform) beschrieben sind, ist im Gegensatz zu den hier beschriebenen Körperchen so beweglich und klein, dass seine Auffindung und Beobachtung schwierig ist.

\section{Erklärung der Abbildungen auf Tafel XX.}

Abbildung 1. Drüsensaft einer Leistendrüse bei secundärer Lues (Alkoholfixation, Giemsa-Färbung).

a Zellen des Drüsenparenchyms.

b Kerntrümmer von Drüsenparenchymzellen.

c Rothe Blutkörperchen.

d Kleine Lymphocyten.

e Grosse Lymphocyten.

f Lymphocytenkern.

g Polynucleärer Leukocyt.

h Spirochaete pallida (Schaudinn-Hoffmann).

1) Studien über Regeneration der Gewebe. Arch. f. mikroskopische Anatonie. Bd. XXIV. S. 81.

2) Zur Kenntniss der Organveränderung bei Leukämie. Diss. Jena 1894.

3) Münch; med. Wochenschr. No. 41. S. 1131.

4) Ueber Blutbefunde bei Syphilis, Masern und Pocken. Medicin. Klinik. 1905. No. 24.

5) Aetiologische Untersuchung über die Syphilis. Verh. der preuss. Akad. der Wissensch. 1905 . 
654 P. Reckzeh, Protoplasmat. Körperchen in d. Lymphdrüsen Syphilitischer.

i Protoplasmatische Körperchen.

k Zerfallsproducte rother Blutkörperchen.

Abbildung 2. a) Schnitt durch eine luetische Lymphdräse (Alkoholhärtung, Vorfärbung in Eosin, Färbung in Thionin, Entfärbung in Formalin) mit 3 Wiakler'schen "tingiblen Kugeln". Nach F. Winkler, "Ueber tingible Kugeln in syphilitischen Producten". Archiv f. Dermatol. u. Syphilis. Bd. 46. S. 3.

b) Doehle's "Protoplasmagebilde" in syphilitischen Producten. Nach P. Doehle, "Ueber Blutbefunde bei Syphilis, Masern und Pocken." Med. Klinik, 1905. No. 24. S. 590. 
Fig 1.

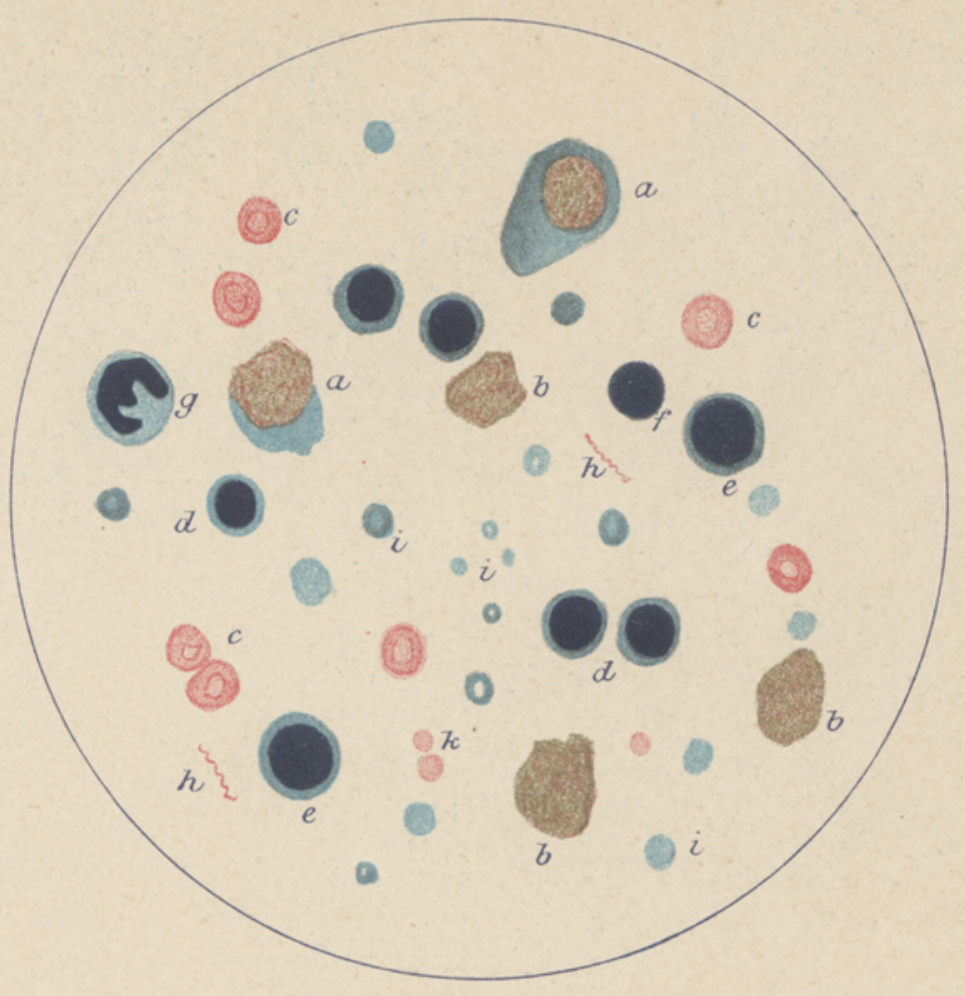

Fig. 2.

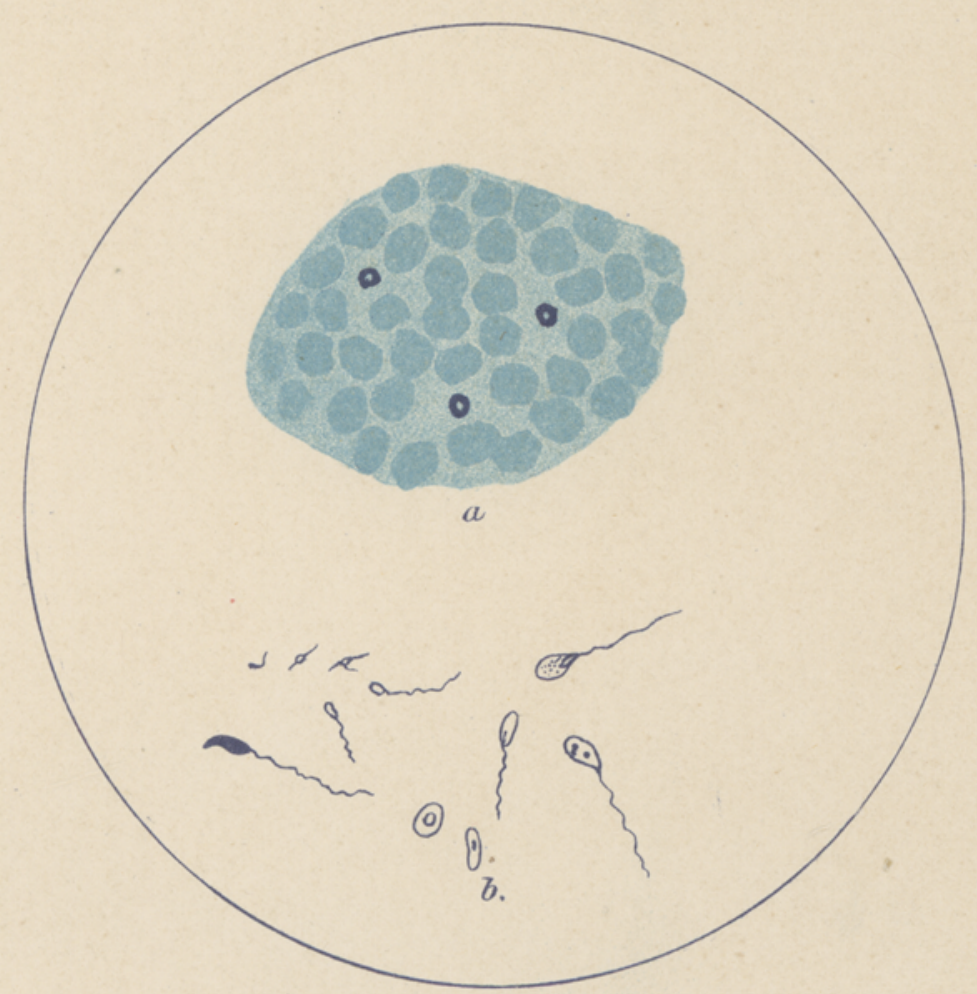

LJThomas, Lith . Frat, Berlin S. 53. 